\title{
Understanding Social-Ecological Systems using Loop Analysis
}

\author{
Marco Scotti ${ }^{1}$ \\ GEOMAR Helmholtz Centre for Ocean Research Kiel, Germany \\ Daniel Filipe da Silva Pereira ${ }^{2}$ \\ GEOMAR Helmholtz Centre for Ocean Research Kiel, Germany \\ Antonio Bodini \\ Department of Chemistry, Life Sciences and Environmental Sustainability, \\ University of Parma, Italy
}

\section{Abstract}

The sustainable management of social-ecological systems (SESs) requires that we understand the complex structure of relationships and feedbacks among ecosystem components and socioeconomic entities. Therefore, the construction and analysis of models integrating ecological and human actors is crucial for describing the functioning of SESs, and qualitative modeling represents an ideal tool since it allows studying dependencies among variables of diverse types. In particular, the qualitative technique of loop analysis yields predictions about how a system's variables respond to stress factors. Different interaction types, scarce information about functional relationships among variables, and uncertainties in the values of the parameters are the rule rather than exceptions when studying SESs. Accordingly, loop analysis seems to be perfectly suitable to investigate them. Here, we introduce the key aspects of loop analysis, discuss its applications to SESs, and suggest it enables making the first steps toward the integration of the three dimensions of sustainability.

Keywords: complex systems, networks, qualitative modeling, social-ecological systems; sustainability

\section{Introduction}

Human societies and their well-being depend on the provision of goods and services from ecosystems (Haines-Young \& Potschin, 2010). Healthy ecosystems respond to human needs by maintaining structure and functioning over time (Costanza \& Mageau, 1999), and the conservation of biodiversity is crucial for preserving

\footnotetext{
1 Corresponding author: marcoscot@gmail.com.

2 Daniel Felipe da Silva Pereira conducted this study at the Department of Chemistry, Life Sciences and Environmental Sustainability, University of Parma, Italy.
} 
stability and productivity of natural systems (Stachowicz et al., 2002; Worm et al., 2006). However, biodiversity is declining worldwide, a trend that raises concerns on the sustainable supply of goods and services from ecosystems (Lotze et al., 2006). The increasing level of human-induced impacts (e.g., overexploitation of resources, introduction of alien species into native environments, chemical pollution, nutrient enrichment, and climate change) threatens biodiversity in both aquatic and terrestrial systems and calls for the formulation of effective conservation practices. Ecological changes are often associated with social and economic transformations that, in turn, reflect their effects back on ecological functions and processes. Discovering and bringing to light these interdependencies requires a shift in focus: from a "within-domain approach" to a global strategy in which the ecosystem as unit of investigation is part of a larger system that embeds socioeconomic dynamics (Hilborn, 2007). Long et al. (2015) identified 15 key principles for implementing ecosystem-based management (EBM). Among these principles, they included the modeling of interconnections between ecological, social, and governance systems, which implies that social-ecological systems (SESs) are networks and that EBM implementation can benefit from the application of the methodologies that network analysis offers.

The network perspective requires that the interactions that link variables belonging to the human and ecological domains are concurrently taken into account, so that the SES as a whole becomes the unit of management. The challenge is the identification of relationships at different hierarchical levels, which occur at various spatial and temporal scales. To facilitate integration, Ostrom (2009) proposed a classificatory framework that describes the four essential dimensions of SESs: resource users, governance system, resource units, and resource system. The relationships among these four dimensions occur at various geographical and temporal scales, within the rules defined by the SES's ecological, social, economic, and political settings. The choice of the suitable scales and the proper identification of the variables that constitute the SES, and their connections, are essential to assess under what conditions sustainability can be enhanced. Moreover, the concept of sustainability is multidimensional and the spatial heterogeneity of SES variables can cause a mismatch between objectives that belong to either the social or ecological domain. This complexity is exemplified by the study of small-scale fisheries in the Mexican state of Baja California Sur, which showed the lack of association between different dimensions of sustainability (Leslie et al., 2015). Policies for the sustainable use of ecosystem goods and services require policy-makers to take into account the set of interactions linking ecological resilience (i.e., the adaptive capacity to withstand recurrent perturbations) to the society, the economy, and the governance rules (Hughes et al., 2005). The integration of these dimensions is challenging and this difficulty is inflated by the adoption of strictly sectoral approaches. Most studies on the social dimension of resources and environmental management focus on social dynamics and treat the ecosystem as a black box; in parallel, the ecological approach 
to sustainability considers the social aspects only at the boundaries of the natural system (Binder et al., 2013; Folke, 2006; Partelow et al., 2019). The balanced integration of social and ecological variables within the same modeling scheme is often precluded by two factors: (1) there are difficulties in the identification of the most important interactions linking the variables; and (2) the mathematical form of interactions is often unknown. Qualitative modeling represents a possible solution to these difficulties. In particular, loop analysis (Levins, 1968, 1974), through its simple application requirements (i.e., describing the presence of links and their signs), can be used to consider the interactions among variables across different domains.

Loop analysis was developed to model the equilibrium levels of a system when growth rates of specific variables are altered by environmental variability. Loop analysis requires that only the sign of the relationship between the variables is specified-that is, whether a variable positively or negatively affects another one. This simplicity overcomes the lack of quantitative information and simplifies the semantic conversion of the concepts related to the processes in which variables take part when belonging to different domains. Most of the works on SESs published so far have focused on ecosystems and considered the human component a source of external perturbations. For example, Bodini et al. (2018) showed how overfishing affected the internal dynamics of the Black Sea, but did not consider how socioeconomic drivers inflated overfishing. Applications of loop analysis to SESs are gaining ground (Dambacher et al., 2007; Martone et al., 2017). Here we show the potential of loop analysis for the integrative modeling of SESs. First, we introduce the methodological aspects behind the tool. Second, we discuss merits and limitations of loop analysis in studying the dynamical behavior of SESs. Then, we compare loop analysis with other qualitative methods that can be applied to SESs. Finally, we present ideas of possible developments that could favor the diffusion of loop analysis in the context of SESs.

\section{Loop analysis: Methodological aspects}

Loop analysis is a qualitative technique for modeling complex systems as signed, directed graphs. Interactions are depicted as either positive or negative effects but their strength is not specified (Figure 1A). Positive interactions are illustrated by arrow-headed links, while negative interactions are visualized with circle-headed links. Any signed digraph has a matrix counterpart (interaction matrix) in which positive (arrowheads) and negative (circle heads) interactions are represented by the coefficients +1 and -1 , respectively. Zeroes in the matrix stand for null direct relationships between any two variables (Figure 1B). The elements along the main diagonal of the interaction matrix are self-effects on the variables and correspond to self-links in the graph (i.e., an arrowhead or circle-head link connecting a variable 
to itself). Loop analysis allows predictions on how the variables will respond to press perturbations that target specific variables. Press perturbations are forces that modify parameters in the rate of change of the variables (Bender et al., 1984), such as environmental warming that enhances the reproductive rate of jellyfish, or ecolabels that increase the rate at which the income of fishing cooperatives is produced. There are as many targets of press perturbations as the number of variables in the system (i.e., any variable can represent the entry point for press perturbations). The effect of press perturbations can be predicted by analyzing the structural properties of the graph (Levins, 1974, 1975).

(A)

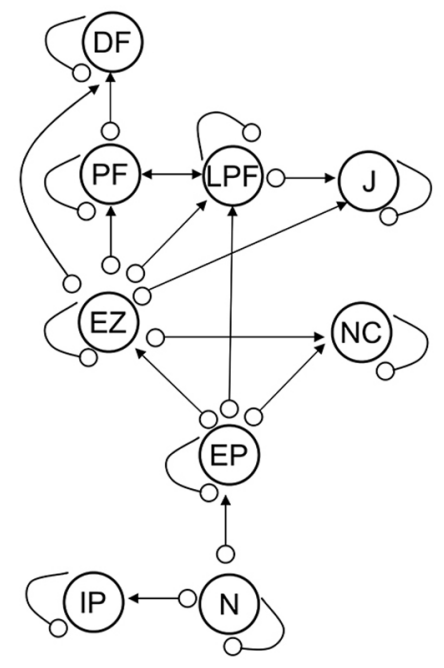

(C)

$\begin{array}{llllllllll} & \mathrm{N} & \mathrm{IP} & \mathrm{EP} & \mathrm{NC} & \mathrm{EZ} & \mathrm{J} & \mathrm{PF} & \mathrm{LPF} & \mathrm{DF} \\ \mathrm{N} & + & + & + & + & - & ?+ & + & + & ?- \\ \mathrm{IP} & - & + & - & - & + & ?- & - & - & ?+ \\ \mathrm{EP} & - & - & + & + & - & ?+ & + & + & ?- \\ \mathrm{NC} & + & + & - & + & ?- & - & ?- & ?- & - \\ \mathrm{EZ} & + & + & - & ?+ & + & + & 0 * & ?- & + \\ \mathrm{J} & ?- & ?- & ?+ & + & ?+ & + & - & - & ?- \\ \mathrm{PE} & - & - & + & - & - & ?- & + & + & ?+ \\ \mathrm{LPE} & ?- & ?- & ?+ & - & - & ?+ & + & + & ?- \\ \mathrm{DE} & 0 * & 0 * & 0 * & + & ?+ & ?- & - & - & +\end{array}$

(B)

$\begin{array}{lrrrllllll} & \mathrm{N} & \text { IP } & \mathrm{EP} & \mathrm{NC} & \mathrm{EZ} & \mathrm{J} & \mathrm{PF} & \mathrm{LPF} & \mathrm{D} \\ \mathrm{N} & -1 & 1 & 1 & 0 & 0 & 0 & 0 & 0 & 0 \\ \mathrm{IP} & -1 & -1 & 0 & 0 & 0 & 0 & 0 & 0 & 0 \\ \mathrm{EP} & -1 & 0 & -1 & 1 & 1 & 0 & 0 & 1 & 0 \\ \mathrm{NC} & 0 & 0 & -1 & -1 & -1 & 0 & 0 & 0 & 0 \\ \mathrm{EZ} & 0 & 0 & -1 & 1 & -1 & 1 & 1 & 1 & 1 \\ \mathrm{~J} & 0 & 0 & 0 & 0 & -1 & -1 & 0 & -1 & 0 \\ \mathrm{PF} & 0 & 0 & 0 & 0 & -1 & 0 & -1 & 1 & 1 \\ \mathrm{LPF} & 0 & 0 & -1 & 0 & -1 & 1 & 1 & -1 & 0 \\ \mathrm{DF} & 0 & 0 & 0 & 0 & -1 & 0 & -1 & 0 & -1\end{array}$

$\mathrm{N}$ - inorganic nutrients

IP - inedible phytoplankton

EP - edible phytoplankton

NC - Noctiluca scintillans

EZ - edible zooplankton

$\mathrm{J}$ - jellyfish

PF - small and medium pelagic fish

LPF - larvae of small and medium pelagic fish

DF - demersal fish

(D)

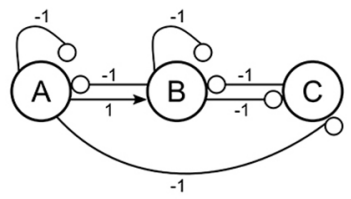

positive input on $A=$ negative effect on $C$ $-1(A-C)+[1 \times(-1)](A-B-C)=-2$

Figure 1. Signed directed graph describing (A) the Black Sea food web and (B) the corresponding matrix of interactions.

Note: In the graph, positive interactions are denoted with arrow-headed links while negative interactions are visualized with circle-headed links. Names of all variables (i.e., nodes in the graph) are below the matrix of interactions. Loop analysis results for the Black Sea in the period 1960-1989 are summarized in the table of predictions (C). Additive and multiplicative rules are considered for predictions (the example here refers to a theoretical system with three variables) (D).

Source: Bodini et al. (2018). 
The links in signed digraphs carry a direction (e.g., where the arrow and the circle point to). By following this direction one can identify paths so that variables that stand far apart from one another in the system can be functionally connected. With reference to Figure 1A, nutrients $(\mathrm{N})$ are connected to demersal fish (DF) by several paths, one of which is: $\mathrm{N} \rightarrow \mathrm{EP} \rightarrow \mathrm{EZ} \rightarrow \mathrm{PF} \rightarrow \mathrm{DF}$. Paths are the routes along which effects of press perturbations travel through the system. Each path carries an effect that is either positive or negative, depending on the product of the signs of the links that compose the path.

Next there is feedback, which can be negative or positive. The former is a process in which an initial change in a variable is reflected back so that its original value is restored. A negative feedback, for example, occurs in agriculture when an increased agricultural yield reduces prices: investments are cut, so that yield is reduced. A positive feedback occurs when an initial change gives rise to a chain of events that amplify the original change. For instance, during the civil war in Colombia the level of violence displaced people from their land, and this contributed to further increases in the level of violence. Since the feedback is a "return effect," it originates when variables are linked by closed paths (i.e., circuits or loops) and its sign is negative or positive depending on the product of the signs of the links that form the loop (see Puccia \& Levins, 1985 for a rigorous method for computing the feedback sign). For example, in Figure 1D the arrow from A to B, and the circle-head link from $\mathrm{B}$ to $\mathrm{A}$, form a closed path, or loop, with negative feedback, because the product of the two links is negative. There can be circuits of different length depending on the number of variables linked together in a closed path. With these definitions we can express conceptually the algorithm of loop analysis (Puccia \& Levins, 1985). The sensitivity of a variable to a press perturbation depends on: (1) whether the perturbation increases (+ sign) or decreases (- sign) the rate of change of the variable through which it enters the system; (2) the sign of the path connecting the variable targeted by the press perturbation to the effect variable (the variable one wants to predict the response of); (3) the sign of the feedback of the subsystem that remains when all variables on the path are ideally removed from the system (this is called complementary feedback); and (4) the overall feedback - that is, the feedback of the circuits that connect all the variables in the system. The algorithm can be summarized in the following formula:

$$
\frac{\partial x_{j}}{\partial c}=\frac{\sum_{i, k}\left[\frac{\partial f_{i}}{\partial c}\right] \times\left[p_{j i}^{(k)}\right] \times\left[F_{n-k}^{(\operatorname{comp})}\right]}{F_{n}}
$$

in which $[\partial f / \partial c]$ expresses whether the rate of change of the target variable $x_{i}$ increases or decreases because of the changing parameter $c$; $\left[p_{j i}{ }^{(k)}\right]$ is the pathway from the target to the response variable; $\left[F_{n-k}^{(\text {comp })}\right]$ is the complementary feedback and $\left[F_{n}\right]$ is the overall feedback. Summation $\left(\sum_{i, k}\right)$ occurs along all paths from the target 
variable $x_{i}$ to the effect variable $x_{j}$. The complementary feedback can be envisioned as a reflecting barrier; if it is negative and strong, the more an impact is reflected back to the effect variable. If it is positive, then the effect variable changes in the opposite direction from the sign of the path. The overall feedback, the denominator of the formula, measures the resistance of the whole system to change. The responses predicted can be positive (+, increase), negative (-, decrease), or null ( 0 , no change), and are summarized in the table of predictions (Figure 1C). The convention is that effects generated by positive perturbations (those increasing the rate of change of the target variables) on row variables can be read along the columns. Consequences of negative perturbations are obtained by reversing the signs of the predictions. Consider a positive press perturbation $\left(\left[\partial f_{i} / \partial c\right]>0\right)$ on node A in Figure 1D: If the focus is on the consequences that the press perturbation has on node $B$, then the path is the positive link from $A$ to $B$ and the complementary subsystem is node $C$ alone; this latter variable does not form any circuit and the complementary feedback is null (i.e., equal to 0 ). The overall feedback is the feedback produced by the circuit that connects all the variables in the system (see Puccia \& Levins, 1985 for further details). In the digraph of Figure 1D there is one single circuit that starts and ends with node $\mathrm{A}$ and connects all variables. This circuit includes one positive and two negative interactions: $\mathrm{A} \rightarrow \mathrm{C}-\circ \mathrm{B}-\circ \mathrm{A}$; its sign is the product of one positive and two negative links and thus it is positive.

In graphs with many variables and interactions, the number of paths between variables often increases, which leads to several ambiguous predictions (i.e., the positive paths counteract negative paths so that a clear sign of the direction of change cannot be identified). To deal with such ambiguities, a simulation approach can be adopted based on a random assignment of strength to each and every link coefficient. To make simulations possible, the signed digraph is transformed in a matrix using +1 to represent positive (arrowhead) links and -1 to indicate negative (circle-head) links. During simulations the coefficient intensities are taken from a uniform distribution in the interval $(0,1]$. This means that the +1 and -1 values in the matrix are substituted by randomly assigned values between $10^{-6}$ (the lower boundary equal to 0 is not included) and 1 while the sign is maintained. Not all the matrices obtained can be used to compute the predictions but only those that satisfy criteria for stability (see Logofet, 1993, for stability conditions matrices must satisfy). According to Bender et al. (1984), the net effect that press perturbations targeting the row variable $x_{i}$ have on the column variable $x_{i}$ are expressed by the elements of the inverse of the matrix that is obtained from the signed digraph (for details see Levins, 1975). After $n$ simulations, an overall table of predictions is constructed by combining the $z$ matrices that are stable and allow matrix inversion. For each stable matrix assembled using simulated interaction strengths, unambiguous responses in the table of predictions are generated (i.e., the signs are certain). The overall table of predictions is composed of symbols that depend on the percentages of signs from the various simulation runs. Hence, if the same entry in the tables of predictions 
from all $z$ (stable) matrices yields the same sign (+ or -$)$ then the expected direction of change is unambiguous. However, during simulations there are cases for which divergent predictions are recorded (i.e., depending on the random arrangement of interaction strengths, the same element in the table of predictions can show either positive or negative sign). The conversion of the outcomes from each simulation run to symbols in the overall table of predictions depends on the percentages of positive $(+)$ and negative (-) signs. The rules to move from simulations results to the overall table of predictions are summarized in Table 1.

Table 1. Rules to convert differences between percentages of signs obtained with simulations (" $\%$ of + " - "\% of -") into predictions (i.e., signs in the overall table of predictions).

\begin{tabular}{|l|l|}
\hline "\% of $+"-$ "\% of $-"$ & Corresponding sign in the table \\
\hline$[-100,-50]$ & - \\
\hline$(-50,-20)$ & $?-$ (tendency to - ) \\
\hline$[-20,20]$ & $0^{*}$ \\
\hline$(20,50)$ & $?+$ (tendency to + ) \\
\hline$[50,100]$ & + \\
\hline $0=100 \%$ & 0 \\
\hline
\end{tabular}

Notes: Round brackets indicate that the extremes are excluded. $0^{*}$ is not a real zero, meaning no changes in the biomass/abundance of variables, but represents neutral results due to relatively balanced amounts of negative and positive effects. When after the complete set of simulations there are entries for which the absence of any effect was always recorded then the symbol in the overall table of predictions is 0 , indicating proper absence of effect (see the last row of the table: $0=100 \%$ ).

Source: Authors' summary.

\section{Merits and limitations of loop analysis to model SESs}

Loop analysis is particularly suitable to investigate SESs. First, interconnections extend beyond the single domains of ecology, economy, and society to create complex networks. For instance, after the Nile perch invaded Lake Victoria a dramatic restructuring of the ecological community took place, which, in turn, cascaded into deep societal and economic changes (Downing et al., 2014). To disentangle drivers and dynamics of change in such a complex scenario, Downing and coworkers designed an eco-social qualitative model that traced connections across disciplinary boundaries. Second, loop analysis educates intuition to cope with complexity. Often, complex systems defy our predictions and effects of policies or management interventions are at best ineffective if not damaging (Levins, 1995). Failure of policies depends on the feedbacks that are produced by the linkages between the variables and that remain hidden to our comprehension if complexity does not become our central intellectual issue. Cinner (2011), 
in discussing problems of reef fishery, emphasizes that the feedback between social and ecological variables may create social-ecological traps (e.g., situations when feedbacks between social and ecological systems lead toward an undesirable state that may be difficult or impossible to reverse). It is extremely interesting to explore these phenomena by loop analysis which, by disentangling feedback loops, helps make the arcane obvious. This capability, however, cannot be fully exploited if the feedback structure of the systems is not adequately represented and the relationships between the variables remain mostly unidirectional; the potential may instead emerge when social and environmental variables are incorporated in a unique model (Dambacher et al., 2007). Third, loop analysis proposes a rigorous approach to diagnosis. Diagnostic approaches are more often requested in the analysis of SESs to causally understand the multiple outcomes that can arise from the interaction of different system attributes (Kittinger et al., 2013). The table of predictions, the main outcome of loop analysis, allows the disentangling of causative mechanisms by linking correlation patterns, sources of change, and network structure (Bodini $\&$ Clerici, 2016; Bodini et al., 2018). For any entry point of press perturbation (any row in the table of predictions, see Figure 1C), variables are predicted to change, so that correlation patterns among them emerge. By comparing such patterns with observed changes in the level of the variables, one can identify which component is affected by external drivers and find the cause and effect mechanisms responsible for those changes due to the linkage structure. Fourth, loop analysis incorporates external drivers as inputs to the rate of change of the variables. External drivers, both social and biophysical, have been described as playing an important role in SES dynamics (Kittinger et al., 2013). For example, in Baja California (Mexico) climate-driven hypoxia caused an excess mortality in marine species with limited mobility, resulting in declines of stocks targeted by small-scale local fisheries, which, in turn, caused small-scale fishers to switch fishing effort toward less-affected species (Micheli et al., 2012). Such effect was explored in a scenario analysis using loop analysis, which predicted large-scale consequences of this external driver (Martone et al., 2017). Fifth, the intuitive visualization of the entities and the interactions among them is suitable for accommodating the general framework proposed by Ostrom (2009) for analyzing the sustainability of SESs. Each node in the digraph can be one of the four elements (i.e., core subsystems: governance system, resource users, resource system, and resource units) and either positive or negative links can visualize their direct relationships. So far the main focus has been dedicated to the visualization of ecological variables and interactions, and the inclusion of socialeconomic aspects has been treated as external to the system (Carey et al., 2014; Espinoza-Tenorio et al., 2013; Reum et al., 2015). Finally, the simple graphical format that constitutes the input for the loop analysis facilitates the participation of all stakeholders to model construction. Although most of the current applications adopted a top-down approach to embed management strategies in models (i.e., literature data were consulted to define the interactions), the study of Espinoza- 
Tenorio et al. (2013) presents a valid alternative. In that work, both quantitative and qualitative information regarding the biological and social aspects of fisheries dynamics and management were retrieved using structured interviews with fishers, participatory research, key informant interviews, and workshops.

Limitations of the methodology should be taken into account. Some limitations have already been discussed (Justus, 2006) and here we focus on those that matter with the use of loop analysis in studying SESs. First, there can be difficulties in defining the timing of changing conditions and that of system response to impacts. SESs are resilient and cope with continuous exposure to press perturbations according to adaptive dynamics principles (Folke, 2006; Hughes et al., 2005). The exact moment at which the system responds to a press perturbation cannot be detected with precision and the contribution of concomitant perturbations may further confound this detection. Second, the variables of SESs (e.g., resources and their users) can show asynchronous behavior and heterogeneous geographical distribution (Leslie et al., 2015). Their optimization does not necessarily occur at the same temporal and spatial scale, an aspect that might remain overlooked when constructing graphs. The uneven geographical distribution of the actors might be addressed by including in the models different variables for the same type of user (e.g., various nodes that indicate the fishers and their interactions in different regions). Third, there can be issues in the identification of the variables exposed to press perturbations (i.e., impacts of overfishing vs climate change). For example, while marketing solutions (e.g., the introduction of ecolabels) can be easily targeted to specific user groups (e.g., the members of fishing cooperatives; see Martone et al., 2017), climate change (e.g., warmer winters) may affect many components of the ecological system with different time of response. One possible solution is prioritizing, as press perturbation targets the most responsive biological variables (e.g., jellyfish have faster blooming rates than expected from the body size; see Nival \& Gorsky, 2001). Finally, loop analysis is problematic for assessing nonlinear relationships. Nonlinearity can emerge by combining the impacts of pathways of different lengths. Longer pathways have lower intensity than shorter ones since the interaction strengths randomly assigned during the simulations are in the interval $(0,1]$ (i.e., the intensity of each pathway is obtained by multiplying the strength of its constitutive links that have upper bound equal to 1). To avoid penalizing the impact of longer pathways, simulations could be carried out by constraining the lower limit from which interaction strengths are randomly drawn during simulations (i.e., by setting the lower limits of some "strong" interactions closer to 1). As an alternative, one could include nonlinear functions to model those specific interactions that play crucial roles for the dynamics of the SES (e.g., by relying on previous literature data or results from specific experiments and surveys). 


\section{Comparison of loop analysis with other qualitative methods for SESs}

SESs form complex networks of linkages and loop analysis is designed to qualitatively predict how variables that are embedded in SESs respond to policies and management interventions (e.g., introduction of new regulations for the exploitation of resources, market-based incentives, and adoption of new marketing solutions; Carey et al., 2014; Dambacher et al., 2007; Levin et al., 2009). A limited number of applications to investigate complex SES have made use of loop analysis, but the interest toward the method has taken little ground in the context of fisheries (Anthony et al., 2013; Carey et al., 2014; Dambacher et al., 2015; Espinoza-Tenorio et al., 2013; Martone et al., 2017). These applications highlight that loop analysis has some potential that extends beyond its limitations. For example, predictions are about the equilibrium level of the variables (Justus, 2006), but real systems are generally not at the equilibrium. However, previous studies have offered evidence that predictions from loop analysis apply successfully to changes in average values of the variables (Bodini, 2000). Average values should be long term, and Bodini et al. (2018) showed that averages taken over either 5 or 10 years can be used to grasp variable responses to press perturbations. The appropriate time scale for taking averages, however, very much depends on the system under investigation.

Other qualitative modeling approaches can be used to study SESs. Fuzzy cognitive maps (FCMs) is one of these. It makes the magnitude of links explicit through a semi-quantification of the relationships that link variables (Kok, 2009; Özesmi \& Özesmi, 2004). The semi-quantification of the links may resolve the ambiguities typical of loop analysis about the net effect generated by the combination of contrasting pathways. Also, FCMs can make predictions about multiple simultaneous perturbations. Both the state of the variables ("concepts," in the technical language of FCMs) and the strength of the links (edges between the concepts) are quantified by assigning standardized values in the range $[0,1]$ for states and $[-1,1]$ for links. Although these are relative values (i.e., each of them is assigned in relation to the others), some criteria for the quantification must be identified. These criteria must be supported by some knowledge about the level of the variables and interactions in the system, and in particular the use of FCMs seems appropriate when the estimates of variable state and link strength are the outcomes of either a combination of multiple FCMs from individual stakeholders or a set of values defined through participatory workshops. The quantification of variables and interactions requires a certain level of knowledge about the system and it automatically selects the working groups among stakeholders that possess some previous understanding of the system under investigation. It follows that FCMs cannot be public, reproducible, and intelligible in the way that loop analysis is. Kok (2009) posits that vague or complex concepts such as "consumer behavior" must not be taken into account when applying FCMs 
because guessing about their magnitude is inherently difficult. On the other hand, loop analysis allows including "awareness" in a malaria model (Yasuoka et al., 2014), "environmental attractiveness" in a tourism model (Bodini et al., 2000), and the "role of the unions" in a model on diabetes (Lewontin \& Levins, 2007). In these aspects it seems that FCMs share one limitation with quantitative models: the tendency to exclude from the analysis factors that are difficult or impossible to measure, no matter if they play a role in the dynamics of the system. The connections among the variables in FCMs are designed on the basis of fuzzy conditional statements ("if ... then") that are of the type "if the level of variable A is high, that of variable B is low." Thus, connections are deduced from correlations between the variables derived from observing the system (Stylios \& Groumpos, 1999). However, Levins and Puccia (1988) point out that patterns of correlation depend on the network structure and the entry point of the perturbation. For example, any two variables may show positive correlation in response to a specific press perturbation but opposite correlation (or no correlation) in response to other press perturbations. It follows that defining interactions between variables on the basis of their correlations may be misleading. In loop analysis, perturbations are variations in parameters that govern the rate of change of variables. For example, a pollutant triggering an increase in the mortality rate of a population. FCMs instead consider the changes in the level of the variables as perturbations. To predict, say, the impacts of a pollutant that affects a population, FCMs consider the reduced abundance induced by the pollutant as the initial event (i.e., the perturbation), on the logical assumption that a toxin, by increasing the mortality of a species, automatically reduces its abundance. Thus, the initial event is deduced from a more or less plausible linear sequence of steps. This series of events overlooks the fact that the response of the target population to the increased mortality is also mediated by the network of interactions with the other variables and that, accordingly, often does not follow commonsense linear expectations. Such assumption leads to the circular argument that FCMs predict the effects given a cause that is in turn an effect that FCMs should predict. On the other hand, we can be confident that the pollutant increases the mortality rate of the target population, which is the initial event in loop analysis. Loop analysis considers the role of the environmental variability in changing the parameters that govern the growth rates of the variables, and does not interpret it solely as the cause of variable fluctuations like FCMs do.

Causal loop diagrams (CLDs; Hanspach et al., 2014; Tenza et al., 2017) and Bayesian belief networks (BBNs; Borsuk et al., 2004; Pollino et al., 2007) have also been applied for analyzing SESs. CLDs make predictions by logically reconstructing the chains of causes and effects between variables on the basis of link polarities (e.g., the signs of the directed links, i.e., the effects of one variable over the other). Predicting the behavior of complex networks by identifying the feedback effects using link polarity (i.e., the effect associated to the link, positive or negative) is difficult and can lead to misleading interpretations (Lane, 2008; Richardson, 1997). 
Most problems originate from polarities. Consider, for example, the case in which the level of violence displaces people from rural areas and forces them to move to the cities (Colombia is a paradigmatic example; see Ibáńez \& Vélez, 2008). The causal connections are that level of violence increases the migration rate (positive link) which, in turn, increases the population level in the city (positive link). Therefore, the expected trend is: the higher the level of violence the greater the increase of urban population. However, if the level of violence gets lower, the migration rate is reduced but this does not reduce the population in the city as it continues to increase unless an opposite migratory flux occurs. Hence, the articulation of causal pathways gets difficult because variables can be both standing stocks and rates of change (Sweeney \& Sterman, 2007). Similarly, specifying the relevant conditional probabilities as required by $\mathrm{BBN}$ can be a laborious and time-consuming process (Marcot et al., 2001; Ticehurst et al., 2007). Moreover, to include feedback mechanisms via cyclic network structures requires dynamic time-explicit BBNs, which depend on extensive parameterization. Similar to FCMs, combining BBNs with loop analysis has great potential for improving predictions and model validation (Anthony et al., 2013; Melbourne-Thomas et al., 2012; Raoux et al., 2018). However, it must be emphasized that these applications of BBNs are based on the signs derived from the analysis of the loop models. As such, their outcomes are contingent on the assumptions and limitations of signed diagraph models.

Central for the understanding of the complex causality in SESs is our ability to individuate what the relevant components of the SESs are and diagram their relationships. The nature of the linkages among these components determines the spreading of the effects through the system and the overall composition of the linkages generates the feedbacks that amplify or buffer such effects. There is no recipe for modeling development but great effort must be devoted to assimilation of facts, observations, and hypotheses. Increasing the reliability of predictions can be possible by designing alternative graphs. This iterative procedure allows addressing uncertainty about the system structure and determining which differences matter. Robust outcomes may be the effect of a core structure common to all models upon which few links added or removed cannot change radically the predictions. The core structure represents the fundamental backbone composed of more certain variables and interactions. Disagreements among stakeholders, scientists, or managers do not limit the application of loop analysis; rather, they offer the opportunity to involve stakeholders in a participatory model construction (Anthony et al., 2013) where different types of system knowledge can be used to determine variables and links that may be important to examine further (Stier et al., 2017). The adoption of such a comprehensive, system-wide approach aims to formulate management strategies that reconcile ecological integrity and intergenerational equity, key dimensions of the sustainable development paradigm. 


\section{Possible developments for the application of loop analysis to SESs}

Making predictions is difficult, especially when we face the uncertainty associated with new, unknown events, changing dynamics, and lack of quantitative data. This is the case, for example, with climate change, which produces completely new phenomena and dynamics. In such context, an adaptive management that allows for continually assessing new evidence has been called for. We believe that in the new scenarios we are facing, the method of loop analysis can be helpful. It has the necessary adaptability to be used in changing contexts: When in doubt about critical linkages and dynamic features, alternative models can be developed to find out which differences matter and to reach robust conclusions. It is also flexible, as it allows including and discarding variables easily, and above all it permits working with variables and links that are not readily measurable, even though their effects are crucial. However, its suitability to investigate SESs can be improved in several ways. The intricacy of the feedbacks can be better resolved if a specific tool for pathways analysis is developed. Returning the total number of pathways, and their strength, between any pair of variables can show how single pathways contribute to specific effects, and which ones are more important in mediating the press perturbations. The question of link strength deserves attention. We specified in the methodological aspects section that link strength is randomly assigned to interaction links during simulations. But this does not contradict the qualitative nature of the method; it only serves to assign certainty to paths and feedbacks in order to get unambiguous predictions, which remain qualitative in nature as only the direction of change for the variables is predicted. Pathway anatomy would help selecting those causal chains that mostly affect system dynamics. Other relevant features that would improve the suitability of loop analysis for modeling SESs include: (1) considering multiple, simultaneous press perturbations; and (2) delimiting upper and lower limits for the strength of interactions. The first point is related to the fact that SESs are often exposed to different types of disturbances whose interplay gives rise to net cumulative responses that would be useful to disentangle (e.g., through the identification of the specific causative chains). Considering multiple press perturbations would greatly contribute to building up an effective diagnostic approach (Kittinger et al., 2013). For example, by loop analysis Bodini et al. (2018) diagnosed that multiple press perturbations, not only overfishing of small and medium pelagic species, were responsible for the restructuring of the Black Sea community during the period 1960-1989. Moreover, socioeconomic drivers amplify the impacts that are triggered by natural processes: for instance, both hypoxia and fisheries management affected abalone stock in the Baja California SES (Martone et al., 2017). The second line of development is conceived to extend loop analysis in a semi-quantitative direction. So far the simulations are performed by randomly sampling interaction strength in the uniform interval $(0,1]$. There are however cases when some interactions are known 
to be either strong or weak; in such cases, varying the strength of these linkages in the whole interval $(0,1]$ does not make sense. However, it is difficult to translate fuzzy concepts such as strong and weak into numbers so that boundaries for the links can be set. In this respect, sensitivity analysis may help. Either the upper (i.e., 1) or the lower (i.e., $10^{-6}$ ) limit of the link magnitude could be iteratively changed so that actual boundaries for certain linkages can be identified through a consistency assessment of the outcomes produced. For example, performing the sensitivity analysis for the strength of specific interactions might help modeling competitive advantages in the ecological domain (e.g., Noctiluca scintillans vs zooplankton in the Black Sea; Bodini et al., 2018) and power or information asymmetries between socioeconomic actors (Bousquet et al., 2015). Therefore, exploring alternative scenarios by constraining the strength of some interactions would be of great benefit for modeling SESs. It should also be noticed that a uniform distribution is considered by default for randomly sampling the strength, but it could be substituted by either normal or skewed (e.g., Poisson) distributions.

\section{Concluding remarks}

The central issue in the study of SESs is to understand interdependencies that cross the boundaries of the classical domains in which scientific and operational knowledge have been settled. Contributions in this respect are expected from tools that allow reconstructing the causal chains that give rise to such interdependencies and that involve variables or components of different nature. For such reconstruction to be effective, tools must overcome the barriers that make communication between domains difficult: (1) the simpler the language used to describe the phenomena and the interactions, the better it is; (2) a lower level of technicality in the algorithms facilitates understanding the outcomes; and (3) flexibility widens the range of applicability to different contexts. Loop analysis shares most of these features: (1) by classifying the interactions in only two categories, positive and negative, it facilitates creating connections between variables that differ dramatically in physical form; (2) the ease by which a model can be constructed as a graph allows keeping up with rapidly changing conditions (i.e., variables and/or links disappear and others become important); (3) the algorithm for predictions refers directly to structural features of the graph and can be visualized, thus making the outcomes easier to understand; and (4) it is also characterized by a wide applicability (often the question of interest is not a particular system but a whole class of systems with some similarity of structure). Furthermore, loop analysis emphasizes the understanding of mechanisms, which is a prime objective when an intellectual and operative framework is taking shape, as in the case of SESs. 


\section{References}

Anthony, K. R., Dambacher, J. M., Walshe, T., \& Beeden, R. (2013). A framework for understanding cumulative impacts, supporting environmental decisions and informing resilience-based management of the Great Barrier Reef World Heritage Area. Australian Institute of Marine Science, CSIRO, NERP Decisions Hub University of Melbourne, and Great Barrier Reef Marine Park Authority. hdl.handle.net/11017/2850

Bender, E. A., Case, T. J., \& Gilpin, M. E. (1984). Perturbation experiments in community ecology: Theory and practice. Ecology, 65(1), 1-13. doi.org/10.2307/1939452

Binder, C. R., Hinkel, J., Bots, P. W. G., \& Pahl-Wostl, C. (2013). Comparison of frameworks for analyzing social-ecological systems. Ecology and Society, 18(4), Article 26. doi.org/ $10.5751 /$ es-05551-180426

Bodini, A. (2000). Reconstructing trophic interactions as a tool for understanding and managing ecosystems: Application to a shallow eutrophic lake. Canadian Journal of Fisheries and Aquatic Sciences, 57(10), 1999-2009. doi.org/10.1139/f00-153

Bodini, A., \& Clerici, N. (2016). Vegetation, herbivores and fires in savanna ecosystems: A network perspective. Ecological Complexity, 28, 36-46. doi.org/10.1016/j.ecocom. 2016.10.001

Bodini, A., Ricci, A., \& Viaroli, P. (2000). A multimethodological approach for the sustainable management of perifluvial wetlands of the Po River (Italy). Environmental Management, 26(1), 59-72. doi.org/10.1007/s002670010071

Bodini, A., Rocchi, M., \& Scotti, M. (2018). Insights into the ecology of the Black Sea through the qualitative loop analysis of the community structure. Limnology and Oceanography, 63(2), 968-984. doi.org/10.1002/lno.10713

Borsuk, M. E., Stow, C. A., \& Reckhow, K. H. (2004). A Bayesian network of eutrophication models for synthesis, prediction, and uncertainty analysis. Ecological Modelling, 173(2-3), 219-239. doi.org/10.1016/j.ecolmodel.2003.08.020

Bousquet, F., Anderies, M., Antona, M., Bassett, T., Benjaminsen, T., Bonato, O., ... Vassal, J.-M. (2015). Socio-ecological theories and empirical research. Comparing social-ecological schools of thoughts in action [Research report]. CIRAD-GREEN. hal.archives-ouvertes.fr/ hal-01130178

Carey, M. P., Levin, P. S., Townsend, H., Minello, T. J., Sutton, G. R., Francis, T. B., ... Ruckelshaus, M. (2014). Characterizing coastal foodwebs with qualitative links to bridge the gap between the theory and the practice of ecosystem-based management. ICES Journal of Marine Science, 71(3), 713-724. doi.org/10.1093/icesjms/fst012

Cinner, J. E. (2011). Social-ecological traps in reef fisheries. Global Environmental Change, 21(3), 835-839. doi.org/10.1016/j.gloenvcha.2011.04.012 
Costanza, R., \& Mageau, M. (1999). What is a healthy ecosystem? Aquatic Ecology, 33(1), 105-115. doi.org/10.1023/A:1009930313242

Dambacher, J. M., Brewer, D. T., Dennis, D. M., Macintyre, M., \& Foale, S. (2007). Qualitative modelling of gold mine impacts on Lihir Island's socioeconomic system and reef-edge fish community. Environmental Science \& Technology, 41(2), 555-562. doi.org/ $10.1021 /$ es0610333

Dambacher, J. M., Rothlisberg, P. C., \& Loneragan, N. R. (2015). Qualitative mathematical models to support ecosystem-based management of Australia’s Northern Prawn Fishery. Ecological Applications, 25(1), 278-298. doi.org/10.1890/13-2030.1

Downing, A. S., van Nes, E. H., Balirwa, J. S., Beuving, J. J., Bwathondi, P. O. J., Chapman, L. J., ... Mooij, W. M. (2014). Coupled human and natural system dynamics as key to the sustainability of Lake Victoria's ecosystem services. Ecology and Society, 19(4), Article 31. doi.org/10.5751/es-06965-190431

Espinoza-Tenorio, A., Wolff, M., Espejel, I., \& Montańo-Moctezuma, G. (2013). Using traditional ecological knowledge to improve holistic fisheries management: Transdisciplinary modeling of a lagoon ecosystem of Southern Mexico. Ecology and Society, 18(2), Article 6. doi.org/10.5751/es-05369-180206

Folke, C. (2006). Resilience: The emergence of a perspective for social-ecological systems analyses. Global Environmental Change, 16(3), 253-267. doi.org/10.1016/j.gloenvcha. 2006.04.002

Haines-Young, R., \& Potschin, M. (2010). The links between biodiversity, ecosystem services and human well-being. In D. G. Raffaelli \& C. L. Frid (Eds.), Ecosystem ecology: A new synthesis (pp. 110-139). Cambridge University Press. doi.org/10.1017/cbo978051175 0458.007

Hanspach, J., Hartel, T., Milcu, A. I., Mikulcak, F., Dorresteijn, I., Loos, J., ... Fischer, J. (2014). A holistic approach to studying social-ecological systems and its application to southern Transylvania. Ecology and Society, 19(4), Article 32. doi.org/10.5751/es-06915190432

Hilborn, R. (2007). Defining success in fisheries and conflicts in objectives. Marine Policy, 31(2), 153-158. doi.org/10.1016/j.marpol.2006.05.014

Hughes, T. P., Bellwood, D. R., Folke, C., Steneck, R. S., \& Wilson, J. (2005). New paradigms for supporting the resilience of marine ecosystems. Trends in Ecology \& Evolution, 20(7), 380-386. doi.org/10.1016/j.tree.2005.03.022

Ibáñez, A. M., \& Vélez, C. E. (2008). Civil conflict and forced migration: The micro determinants and welfare losses of displacement in Colombia. World Development, 36(4), 659-676. doi.org/10.1016/j.worlddev.2007.04.013

Justus, J. (2006). Loop analysis and qualitative modeling: Limitations and merits. Biology and Philosophy, 21(5), 647-666. doi.org/10.1007/s10539-006-9050-x 
Kittinger, J. N., Finkbeiner, E. M., Ban, N. C., Broad, K., Carr, M. H., Cinner, J. E., ... Crowder, L. B. (2013). Emerging frontiers in social-ecological systems research for sustainability of small-scale fisheries. Current Opinion on Environmental Sustainability, 5(3-4), 352-357. doi.org/10.1016/j.cosust.2013.06.008

Kok, K. (2009). The potential of fuzzy cognitive maps for semi-quantitative scenario development, with an example from Brazil. Global Environmental Change, 19(1), 122133. doi.org/10.1016/j.gloenvcha.2008.08.003

Lane, D. C. (2008). The emergence and use of diagramming in system dynamics: A critical account. Systems Research and Behavioral Science, 25(1), 3-23. doi.org/10.1002/sres.826

Leslie, H. M., Basurto, X., Nenadovic, M., Sievanen, L., Cavanaugh, K. C., Cota-Nieto, J. J., ... Aburto-Oropeza, O. (2015). Operationalizing the social-ecological systems framework to assess sustainability. Proceedings of the National Academy of Sciences of the USA, 112(19), 5979-5984. doi.org/10.1073/pnas.1414640112

Levin, P. S., Fogarty, M. J., Murawski, S. A., \& Fluharty, D. (2009). Integrated ecosystem assessments: Developing the scientific basis for ecosystem-based management of the ocean. PLoS Biology, 7(1), e1000014. doi.org/10.1371/journal.pbio.1000014

Levins, R. (1968). Evolution in changing environments: Some theoretical explorations. Princeton University Press.

Levins, R. (1974). Discussion paper: The qualitative analysis of partially specified systems. Annals of the New York Academy of Sciences, 231(1), 123-138. doi.org/10.1111/j.17496632.1974.tb20562.x

Levins, R. (1975). Evolution in communities near equilibrium. In M. L. Cody and J. M. Diamond (Eds.), Ecology and evolution of communities (pp. 16-50). Harvard University Press.

Levins, R. (1995). Preparing for uncertainty. Ecosystem Health, 1(1), 47-57.

Levins, R., \& Puccia, C. J. (1988). The controlling factor in biological communities. Coenoses, 3(3), 149-154. www.jstor.org/stable/43460977

Lewontin, R. C., \& Levins, R. (2007). Educating the intuition to cope with complexity. In R. C. Lewontin \& R. Levins (Eds.), Biology under the influence: Dialectical essays on ecology, agriculture, and health (pp. 183-198). Monthly Review Press.

Logofet, D. (1993). Matrices and graphs: Stability problems in mathematical ecology. CRC Press.

Long, R. D., Charles, A., \& Stephenson, R. L. (2015). Key principles of marine ecosystembased management. Marine Policy, 57, 53-60. doi.org/10.1016/j.marpol.2015.01.013

Lotze, H. K., Lenihan, H. S., Bourque, B. J., Bradbury, R. H., Cooke, R. G., Kay, M. C., ... Jackson, J. B. (2006). Depletion, degradation, and recovery potential of estuaries and coastal seas. Science, 312(5781), 1806-1809. doi.org/10.1126/science.1128035 
Marcot, B. G., Holthausen, R. S., Raphael, M. G., Rowland, M. M., \& Wisdom, M. J. (2001). Using Bayesian belief networks to evaluate fish and wildlife population viability under land management alternatives from an environmental impact statement. Forest Ecology and Management, 153(1-3), 29-42. doi.org/10.1016/s0378-1127(01)00452-2

Martone, R. G., Bodini, A., \& Micheli, F. (2017). Identifying potential consequences of natural perturbations and management decisions on a coastal fishery social-ecological system using qualitative loop analysis. Ecology and Society, 22(1), Article 34. doi.org/ $10.5751 /$ es-08825-220134

Melbourne-Thomas, J., Wotherspoon, S., Raymond, B., \& Constable, A. (2012). Comprehensive evaluation of model uncertainty in qualitative network analyses. Ecological Monographs, 82(4), 505-519. doi.org/10.1890/12-0207.1

Micheli, F., Saenz-Arroyo, A., Greenley, A., Vazquez, L., Espinoza Montes, J. A., Rossetto, M., \& De Leo, G. A. (2012). Evidence that marine reserves enhance resilience to climatic impacts. PLoS ONE, 7, e40832. doi.org/10.1371/journal.pone.0040832

Nival, P., \& Gorsky, G. (2001). Conditions for blooming of pelagic species. In CIESM Workshop Monographs, No. 14-Gelatinous zooplankton outbreaks: Theory and practice (pp. 23-27). Commission Internationale pour l'Exploration Scientifique de la Méditerranée (CIESM [Mediterranean Science Commission]).

Ostrom, E. (2009). A general framework for analyzing sustainability of social-ecological systems. Science, 325(5939), 419-422. doi.org/10.1126/science.1172133

Özesmi, U., \& Özesmi, S. L. (2004). Ecological models based on people's knowledge: A multi-step fuzzy cognitive mapping approach. Ecological Modelling, 176(1-2), 43-64. doi.org/10.1016/j.ecolmodel.2003.10.027

Partelow, S., Fujitani, M., Soundararajan, V., \& Schlüter, A. (2019). Transforming the social-ecological systems framework into a knowledge exchange and deliberation tool for comanagement. Ecology and Society, 24(1), Article 15. doi.org/10.5751/es-10724-240115

Pollino, C. A., Woodberry, O., Nicholson, A., Korb, K., \& Hart, B. T. (2007). Parameterisation and evaluation of a Bayesian network for use in an ecological risk assessment. Environmental Modelling \& Software, 22(8), 1140-1152. doi.org/10.1016/j. envsoft.2006.03.006

Puccia, C. J., \& Levins, R. (1985). Qualitative modeling of complex systems: An introduction to loop analysis and time averaging. Harvard University Press.

Raoux, A., Dambacher, J. M., Pezy, J. P., Mazé, C., Dauvin, J. C., \& Niquil, N. (2018). Assessing cumulative socio-ecological impacts of offshore wind farm development in the Bay of Seine (English Channel). Marine Policy, 89, 11-20. doi.org/10.1016/j.marpol. 2017.12.007

Reum, J. C., McDonald, P. S., Ferriss, B. E., Farrell, D. M., Harvey, C. J., \& Levin, P. S. (2015). Qualitative network models in support of ecosystem approaches to bivalve aquaculture. ICES Journal of Marine Science, 72(8), 2278-2288. doi.org/10.1093/icesjms/fsv119 
Richardson, G. P. (1997). Problems in causal loop diagrams revisited. System Dynamics Review, 13(3), 247-252. doi.org/10.1002/(sici)1099-1727(199723)13:3<247::aid-sdr $128>3.0$. co; $2-9$

Stachowicz, J. J., Fried, H., Osman, R. W., \& Whitlatch, R. B. (2002). Biodiversity, invasion resistance, and marine ecosystem function: Reconciling pattern and process. Ecology, 83(9), 2575-2590. doi.org/10.1890/0012-9658(2002)083[2575:birame]2.0.co;2

Stier, A. C., Samhouri, J. F., Gray, S., Martone, R. G., Mach, M. E., Halpern, B. S., ... Levin, P. S. (2017). Integrating expert perceptions into food web conservation and management. Conservation Letters, 10(1), 67-76. doi.org/10.1111/conl.12245

Stylios, C. D., \& Groumpos, P. P. (1999). A soft computing approach for modelling the supervisor of manufacturing systems. Journal of Intelligent and Robotic Systems, 26(3-4), 389-403. doi.org/10.1023/A:1008165916707

Sweeney, L. B., \& Sterman, J. (2007). Thinking about systems: Student and teacher conceptions of natural and social systems. System Dynamics Review, 23(2-3), 285-311. doi.org/10.1002/sdr.366

Tenza, A., Pérez, I., Martínez-Fernández, J., \& Giménez, A. (2017). Understanding the decline and resilience loss of a long-lived social-ecological system: Insights from system dynamics. Ecology and Society, 22(2), Article 15. doi.org/10.5751/es-09176-220215

Ticehurst, J. L., Newham, L. T., Rissik, D., Letcher, R. A., \& Jakeman, A. J. (2007). A Bayesian network approach for assessing the sustainability of coastal lakes in New South Wales, Australia. Environmental Modelling \& Software, 22(8), 1129-1139. doi.org/10.1016/ j.envsoft.2006.03.003

Worm, B., Barbier, E. B., Beaumont, N., Duffy, J. E., Folke, C., Halpern, B. S., ... Watson, R. (2006). Impacts of biodiversity loss on ocean ecosystem services. Science, 314(5800), 787-790. doi.org/10.1126/science.1132294

Yasuoka, J., Jimba, M., \& Levins, R. (2014). Application of loop analysis for evaluation of malaria control interventions. Malaria Journal, 13, Article 140. doi.org/10.1186/14752875-13-140 
This text is taken from Human Ecology Review, Volume 26, Number 2, 2020, published by ANU Press, The Australian National University, Canberra, Australia. doi.org/10.22459/HER.26.02.2020.03 